\title{
Observational study of colonoscopy techniques used for acute colorectal obstruction: A single-center experience
}

\author{
QIAN CHEN $^{1}$, QIN YU ${ }^{2}$, JING-JING YU ${ }^{2}$, MEI LIU $^{1}$, HUA-PING XIE $^{1}$, \\ BIN CHENG $^{1}$, QIAO-ZHEN GUAO ${ }^{1}$, GUANG-QUAN LIAO ${ }^{1}$ and HUA QIN ${ }^{1}$
}

${ }^{1}$ Endoscopic Unit, Department of Gastroenterology and Hepatology; ${ }^{2}$ Hepatic Surgery Centre, Tongji Hospital, Tongji Medical College, Huazhong University of Science and Technology (HUST), Wuhan, Hubei 430030, P.R. China

Received October 3, 2016; Accepted December 9, 2016

DOI: $10.3892 / \mathrm{mco} .2017 .1133$

\begin{abstract}
The prevalence of acute colorectal obstruction at the hospital setting is high. There is need for improvement in the quality of colonoscopy for relieving obstruction. A retrospective, uncontrolled, open-label study was conducted, with the aim of documenting the causes of acute colorectal obstruction and the quality of colonoscopy practice in managing obstruction at a university hospital in China. A total of 61 adult patients with acute colorectal obstruction treated with urgent colonoscopy between February, 2011 and January, 2016 were identified at the Endoscopic Unit of Tongji Hospital (Wuhan, China). The technique success rate was the primary outcome. The etiology was mainly related to malignant diseases, including colorectal cancer in 53 patients $(86.9 \%)$ and advanced peritoneal serous carcinoma leading to extrinsic colonic compression in 1 patient (1.6\%). Benign causes of obstruction included foreign bodies in 3 patients $(4.9 \%)$ and fecal impaction in 2 patients (3.3\%); furthermore, anastomotic strictures were found in 2 patients $(3.3 \%)$. The technique success rate was $98.4 \%$ (60/61). All colorectal cancer patients who underwent urgent colonoscopy were admitted to the Department of Surgery for placement of a self-expanding metallic stent; 1 patient who had an extracolonic malignancy underwent placement of a transanal colonoscopic decompression tube as palliative therapy and 2 patients with anastomotic strictures underwent endoscopic balloon dilation. Furthermore, colonoscopy was used for extracting the foreign body, as well as removing fecal stones in all the patients. In conclusion, urgent colonoscopy as a minimal invasive approach is associated with certain advantages for diagnosing and managing acute colorectal
\end{abstract}

Correspondence to: Professor Hua Qin, Endoscopic Unit, Department of Gastroenterology and Hepatology, Tongji Hospital, Tongji Medical College, Huazhong University of Science and Technology (HUST), 1095 Jie Fang Avenue, Wuhan, Hubei 430030, P.R. China

E-mail: drqinhua@163.com; c_q_n@yahoo.com

Key words: acute colorectal obstruction, foreign body, self-expanding metallic stent, transanal colonoscopic decompression tube, endoscopic balloon dilation obstruction. Furthermore, experienced operators achieved a better outcome when managing colorectal obstruction.

\section{Introduction}

Acute colorectal obstruction is an emergency condition that requires early identification and intervention. In addition to recognizing the common etiological factors responsible for the obstruction, physicians must maintain a high index of suspicion for complete obstruction, gangrenous or perforated bowel, which should be referred for surgical consultation. For partial colorectal obstruction, a less invasive approach is desirable to reduce the risk of adverse outcomes associated with emergency surgery. Applying urgent colonoscopy allows for direct evaluation of the site of obstruction and subsequent determination of the cause of obstruction and provision of treatment $(1,2)$. For example, although foreign body impaction in the colon is not common in everyday emergency practice compared with upper gastrointestinal (GI) foreign body ingestion, the value of urgent colonoscopy for identifying a foreign body appears to be more efficient compared with imaging studies, such as plain abdominal X-ray or computed tomography $(3,4)$. Fecal impaction is a common GI disorder that may lead to acute complications and it presents with symptoms similar to those observed in colon obstruction, such as abdominal pain and distention, nausea, vomiting and anorexia (5). Congenital and acquired conditions of the colon and rectum, including Hirschsprung's disease and Chagas' disease, may cause fecal impaction. Anatomic and functional abnormalities of the anorectum should also be considered and excluded (5). Therefore, direct visualization by colonoscopy may also be a useful tool for diagnosing and treating fecal impaction.

Acute malignant colonic obstruction is common in the hospital setting. Up to $29 \%$ of patients with advanced colorectal cancer present with acute colonic obstruction from intraluminal tumor growth. By contrast, metastatic or invasive non-colorectal cancer may lead to acute obstruction by extrinsic compression (6). Treatment options include transanal colonoscopic decompression tubes and placement of a self-expanding metallic stent (SEMS), which is primarily used for malignant obstruction, while endoscopic balloon dilation is used for benign indications, such as strictures resulting from surgical anastomosis and inflammatory bowel disease (1). 
The application of SEMS has recently become more frequent for the palliation of metastatic colorectal cancer and as a bridge to surgery for obstructing tumors, despite the concerns regarding safety and efficacy in recent systematic reviews and meta-analyses (6,7). Additional considerable variations in practice may exist due to the lack of expertise and technical difficulties $(8,9)$.

SEMS placement involves passing a guidewire through the obstruction. The wire is manipulated with either fluoroscopy alone, as in the over-the-wire (OTW) method, or combined with endoscopic visualization, as in the through-the-scope (TTS) technique $(8,10)$. In the literature, it appears that experienced operators using TTS achieved better technical and clinical outcomes $(8,9,11,12)$. The present study demonstrated that TTS is a practical tool for effectively resolving bowel obstruction in our unit. In addition, in a case with near complete obstruction, in which the slim endoscope could not be passed through the lesion, delivery of SEMS was guided by real-time radiography and $\mathrm{CO}_{2}$ gas was used as a contrast agent, allowing assessment of the morphology and length of the obstruction.

\section{Patients and methods}

Single endoscopy unit study. A database of all patients treated between February 1, 2011 and January 31, 2016 at the the Endoscopy Unit of Tongji Hospital (Wuhan, China) was accessed. Tongji Hospital is a state-owned teaching hospital. The Department of Internal Medicine and its Endoscopy Unit is a referral center for Gastroenterology and Hepatology and has availability of all endoscopic facilities and treatment modalities for diagnostic, therapeutic and palliative endoscopy. During the study period, 43,732 esophagogastroduodenoscopies were performed annually at this Unit.

Retrospective analysis. For the purposes of this study, the Endoscopy Unit records were retrospectively reviewed, as were hospital medical records. Approval for the study was granted by the Institutional Ethics Committee. A total of 61 patients from the inpatient care who underwent emergency colonoscopy management were evaluated by both the surgeons and endoscopists prior to the procedure. All the patients exhibited clinical characteristics of colorectal obstruction, such as obstipation or constipation for $>48 \mathrm{~h}$, abdominal distension, nausea, vomiting, or cramping abdominal pain. The abdominal X-rays revealed colon dilation proximal to the obstruction site, and large-bowel obstruction was confirmed by means of either computed tomography (CT), barium enema, or colonoscopy.

Technique. The primary indication of SEMS insertion is intended to be a bridge to surgery for obstructive colorectal cancer (1). For elderly patients with unresectable malignancies who have severe medical comorbidities and are in a poor overall condition, a transanal colonoscopic decompression tube may be placed. The indication for endoscopic balloon dilation is treatment of strictures associated with surgical anastomoses.

Procedure for SEMS placement. All the SEMS placement procedures were performed by one of four experienced endoscopists using the TTS technique. The authors used the following procedures for stenting: First, a thin gastroscope (GIF XP260; Olympus, Tokyo, Japan) was passed through the stricture. The stricture length was estimated while monitoring the endoscope advancing through the stenosis site. Second, a biliary guidewire (Jagwire; diameter 0.035 inches, length $450 \mathrm{~cm}$; Boston Scientific, Hemel Hempstead, UK) was inserted into the working channel $(2.0 \mathrm{~mm}$ in diameter) of the endoscope (GIF XP260; Olympus) to pass through the obstruction for $\sim 20 \mathrm{~cm}$ or more, to prevent it from slipping back through the obstruction. Third, the guidewire was secured by an assistant as the endoscope was retracted. During retraction, the endoscopist measured the tumor length and the proximal and distal extension, which was crucial for subsequent correct stent placement. Fourth, the stent length was then selected according to the length of the tumor, with a deployed length of $\geq 2 \mathrm{~cm}$ longer compared with that of the stricture. The SEMS delivery system was placed over the guidewire followed by re-intubating with the endoscope proximal to the stricture, allowing accurate SEMS positioning under direct vision by aligning the upper end of the stent beyond the tumor margin for $\sim 1 \mathrm{~cm}$. The stent was then slowly deployed from its distal to its proximal end, maintaining proximal traction on the stent introducer to prevent distal migration.

In a case with near complete obstruction, in which the slim endoscope could not pass through, delivery of SEMS was guided by real-time radiography and $\mathrm{CO}_{2}$ gas was used as a contrast agent, allowing assessment of the morphology and length of the obstruction. Radiographic visualization and an immediate stool flush were used to assess the patency after completion of stent placement.

All SEMSs used were uncovered metal mesh devices from MicroTech (diameter, $20 \mathrm{~mm}$; length, 60-100 mm; MicroTech Co., Nanjing, China). The median procedure time was $30 \mathrm{~min}$ (range, 20-60 min).

\section{Results}

Etiology. Urgent endoscopic management of acute colorectal obstruction is a rapidly developing field at the Tongji Endoscopy Unit. In this retrospective study, 61 patients with acute colorectal obstruction were identified between February, 2011 and January, 2016. As shown in Fig. 1, the number of cases increased each year, with only 4 cases in the first year and 28 cases in the fifth year (Fig. 1). The etiological factors of obstruction included colorectal cancer (53/61), extracolonic malignancy, namely advanced peritoneal serous carcinoma $(1 / 61)$, foreign bodies (3/61), fecal impaction (2/61) and anastomotic strictures (2/61; Table I).

Benign colorectal obstruction. Three cases were caused by foreign body obstruction. The average age was 39.7 years and all the patients were male. In two cases the foreign objects had been intentionally inserted into the rectum. The objects recovered from the rectum included a sausage and cotton tissue (Fig. 2), whereas 1 patient had previously undergone sigmoid colectomy and a piece of surgical gauze was retained in the distal loop. A retrieval device (alligator forceps) was successfully used to extract the foreign bodies.

Two cases were caused by fecal impaction: One was a 48-year-old female patient who had chronic constipation and 
Table I. Etiology and techniques used for acute colorectal obstruction.

\begin{tabular}{lrl}
\hline Etiology & No. of patients & Techniques \\
\hline $\begin{array}{l}\text { Malignant conditions } \\
\text { Colorectal cancer }\end{array}$ & 53 & SEMS \\
$\begin{array}{l}\text { Advanced peritoneal serous cancer } \\
\text { Benign conditions } \\
\text { Foreign bodies (food, cotton tissue } \\
\text { and surgical gauze) }\end{array}$ & 3 & Forceps method \\
$\begin{array}{l}\text { Fecal impaction (chronic constipation, } \\
\text { anatomic anorectal abnormalities) } \\
\text { Anastomotic strictures (post-rectal cancer) }\end{array}$ & 2 & Snare method \\
\hline
\end{tabular}

SEMS, self-expanding metallic stent.

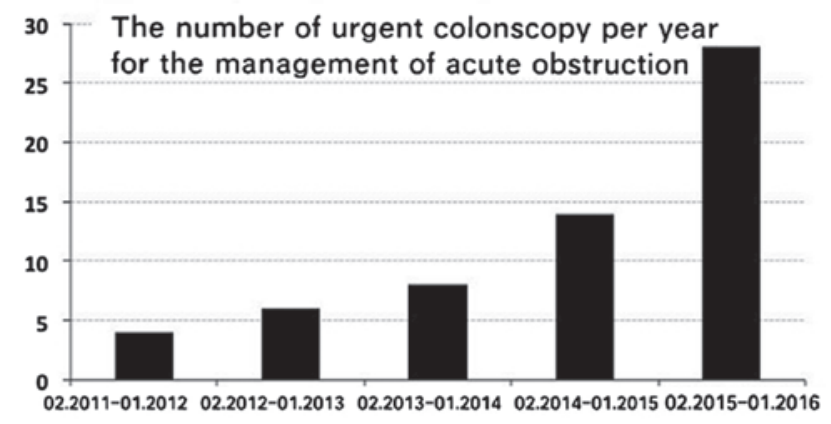

Figure 1. Number of urgent colonoscopies per year for the management of acute colorectal obstruction at the Endoscopy Unit of Tongji Hospital. A total of 61 patients with acute obstruction underwent the procedure. There were 4 cases between February, 2011 and January, 2012, 6 between February, 2012 and January, 2013, 8 between February, 2013 and January, 2014, 14 between February, 2013 and January, 2014, and 28 cases between February, 2015 and January, 2016.

the hardened stool was impacted in her descending colon close to the splenic fixture, with subsequent obstruction. The other case was a 28-year-old female patient who suffered from congenital vaginal obstruction and had undergone vaginal reconstruction with a sigmoid colon segment when she was 23 years old. The patient subsequently developed chronic constipation, and colonoscopy revealed fecal impaction in the sigmoid colon. In both cases, the snare method was used to break up the stone-like mass, which was then fragmentarily removed.

Malignant colorectal obstruction. In our unit, SEMS placement is emergently applied as a bridge to surgery. A total of 53 such patients were identified, 34 men (64.2\%) and 19 women (35.8\%), aged $19-82$ years, with a mean age of 56 years at the time of the procedure. The most frequent stricture location was the sigmoid in 21 patients, followed by the rectosigmoid junction (11 patients), the rectum (10 patients), the descending colon (5 patients), the splenic fixture (5 patients) and the transverse colon (1 patient) (Table II).

Initial technical success (defined as the ability to adequately place a stent across the site of obstruction) was achieved in
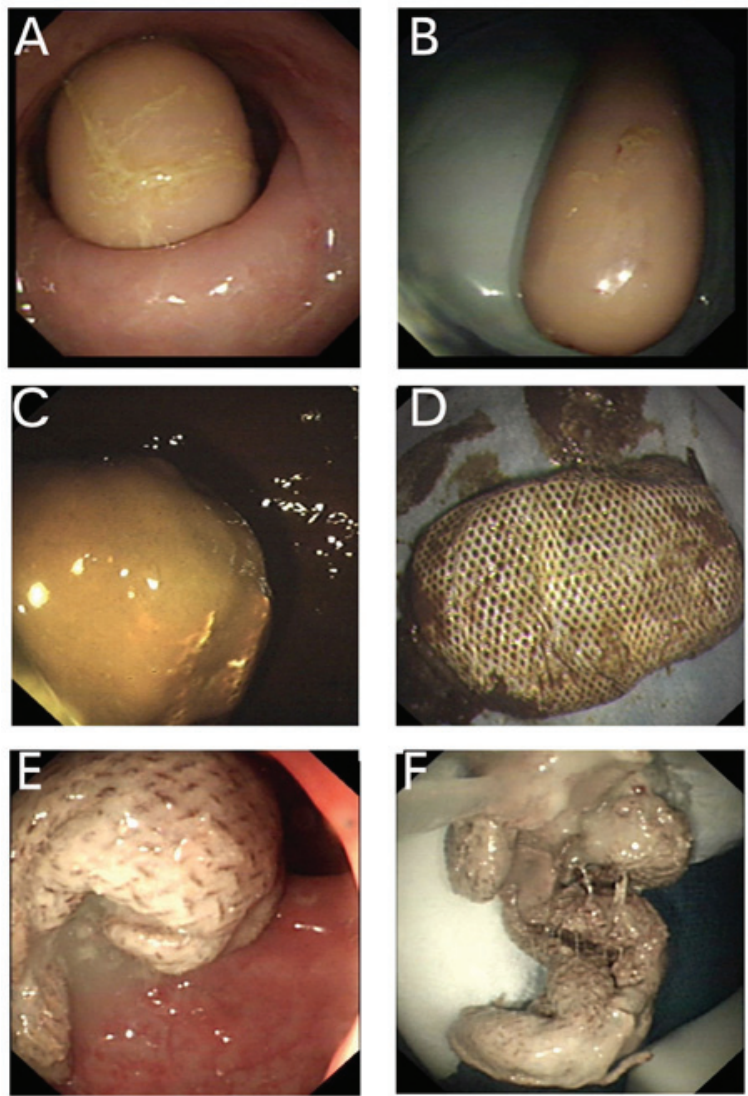

Figure 2. Cases with obstruction due to foreign bodies. In 3 patients who underwent colonoscopy, foreign bodies were identified, including (A and B) a sausage, $(C$ and $D)$ cotton tissue and (E and F) surgical gauze. A retrieval device (alligator forceps) was successfully used to extract the foreign bodies. (A, C and D) Endoscopic view of the objects that were impacted in the colon. (B, D and F) Objects following removal from the colon.

52 of the 53 cases $(98 \%)$. In 1 patient with sigmoid colon cancer, the stent could not be passed through the stricture after delivering the guidewire under direct vision; the patient subsequently underwent palliative surgery, with complete relief of the obstruction. Clinical success (defined as relief of obstruction with the passage of stool and gas) was achieved in 

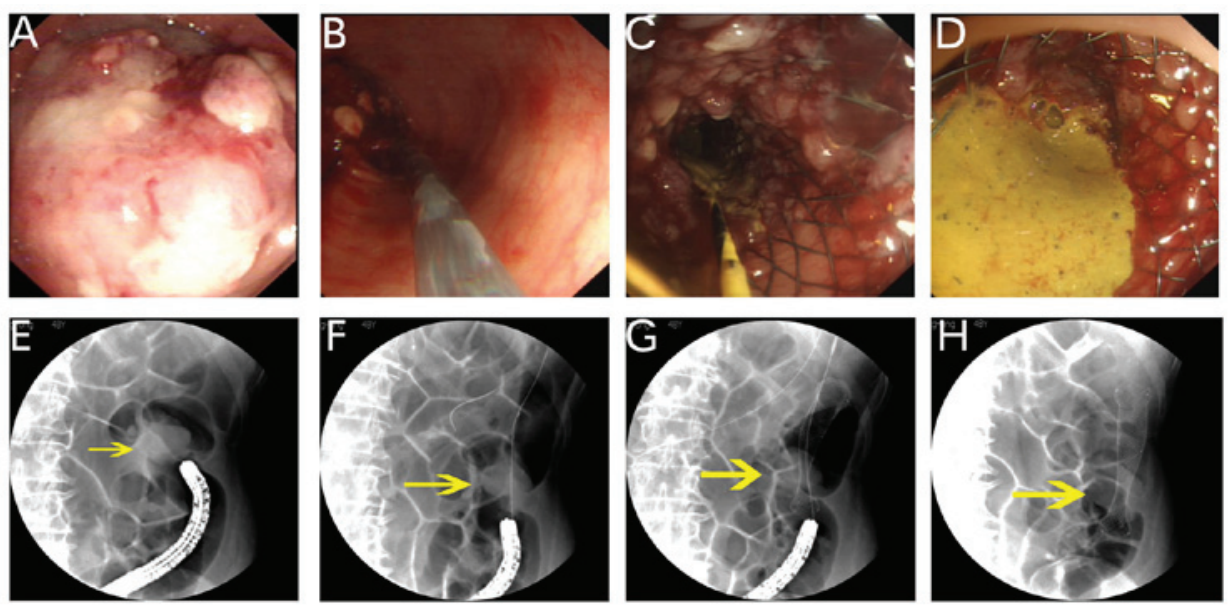

Figure 3. Placement of SEMS using the over-the-wire technique in the descending colon. (A) Endoscopic image showing the site of complete obstruction by malignancy. (B and C) The pre-deployed SEMS was advanced across the lesion under guidewire guidance and then fully deployed. (D) The guidewire was withdrawn and stool was passed after SEMS deployment. (E-H) Radiographic views at time of the endoscopic procedure. (E) The tumor was outlined and indicated by the yellow arrow. (F) The biliary guidewire was inserted into the working channel of the endoscope under radiographic guidance to pass through the obstruction for $\sim 20 \mathrm{~cm}$. (G) The SEMS was advanced across the lesion under guidewire and radiographic guidance. (H) The stent was deployed and the guidewire was withdrawn. SEMS, self-expanding metallic stent.
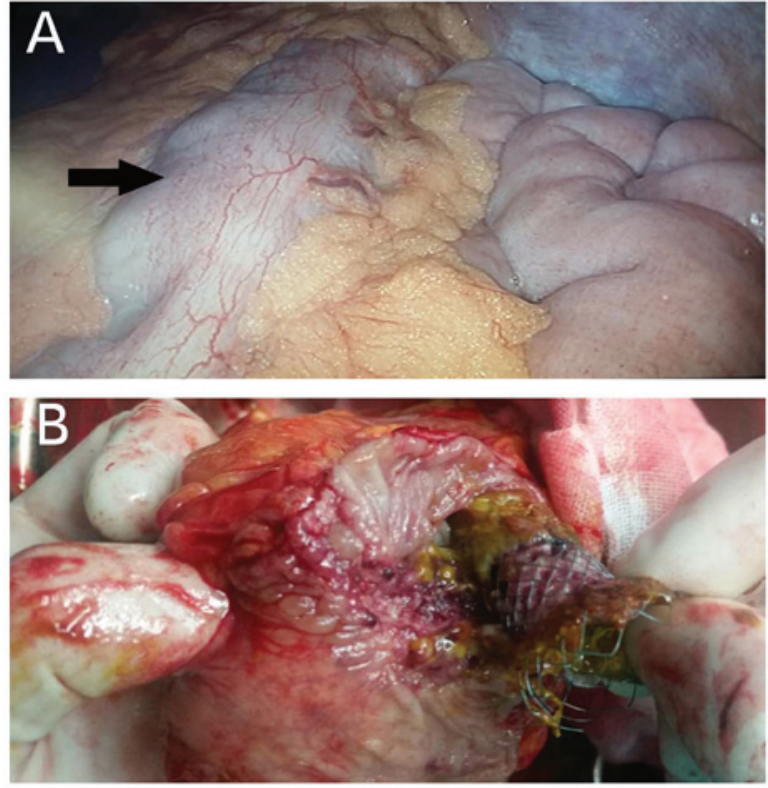

Figure 4. Laparoscopy 3 days after stent placement for acute malignant colon obstruction. A one-stage operation was performed. (A) The target colon (arrow) was mobilized laparoscopically. (B) Gross photograph of the resected colon with the tumor containing a preoperatively placed SEMS. SEMS, self-expanding metallic stent.

all 52 patients $(100 \%)$ within $24 \mathrm{~h}$, although in $~ 95 \%$ patients bowel decompression started immediately after stent deployment. There were no procedure-related complications, such as perforation or stent dislocation. All the patients underwent surgery at 3-7 days following SEMS placement. Importantly, stenting gained time for staging, treatment planning, neoadjuvant therapies and patient optimization. Of these patients, $8(15.3 \%)$ were found to have distant organ metastases at staging and, hence, avoided unnecessary major surgery.

In a 64-year-old female patient with near complete obstruction of the descending colon due to malignancy, passing a guidewire and subsequently placing a stent were performed by an endoscopist experienced in endoscopic retrograde cholangiopancreatography (ERCP; 264 procedures annually). Radiography was used at the time of procedure to monitor traversing the stricture and to ensure complete patency of the prosthesis following stent placement (Fig. 3). The patient underwent laparotomic surgery 3 days later and the SEMS was removed (Fig. 4). A one-stage operation was achieved.

In an 85-year-old female patient who had extraluminal compression resulting from advanced peritoneal serous carcinoma, a transanal colonic decompression tube was placed in the sigmoid colon as palliative therapy. For benign obstructions resulting from surgical anastomoses, endoscopic balloon dilation of the strictures was successfully applied in 2 patients, without complications.

\section{Discussion}

Patients admitted to Tongji Hospital with acute colon obstruction were readily examined by the general/trauma surgeons. Patients with severe unremitting pain or peritoneal signs suspected to have complete obstruction, are generally referred for surgical consultation. The decision to perform urgent colonoscopy is based on the patient's condition, in addition to excluding the potential risk of perforation. Following further evaluation by endoscopists, these patients are immediately prepared for endoscopy. The incidence in our population of patients requiring an urgent colonoscopic procedure was $0.56 \%$.

Urgent colonoscopy is widely used to relieve obstruction resulting from benign conditions, such as volvulus, inflammatory bowel disease, diverticulitis, anastomotic strictures, radiation injury, ischemia, foreign bodies and intussusception 1). Balloon dilation is a well-established technique for treating strictures resulting from surgical anastomoses and inflammatory bowel disease (13-15). However, our experience is limited, compared with our experience with dilating esophageal strictures. One reason is that the majority of 
Table II. SEMS patient demographics, method of stenting and experience of the operator.

\begin{tabular}{lc}
\hline Variables & $\mathrm{N}(\%)$ \\
\hline Age, years [mean (range)] & $56(19-82)$ \\
Gender & \\
Male & $34(64.2)$ \\
Female & $19(35.8)$ \\
Reason for stenting & \\
Bridge to CRC surgery & $8(15.4)$ \\
Palliative for CRC & $44(84.6)$ \\
Site & \\
Rectum & $10(18.9)$ \\
Sigmoid & $21(39.6)$ \\
Rectosigmoid junction & $11(20.8)$ \\
Descending colon & $5(9.4)$ \\
Splenic fixture & $5(9.4)$ \\
Transverse colon & $1(1.9)$ \\
SEMS used & \\
1 & $52(98.1)$ \\
$0^{a}$ & $1(2.9)$ \\
SEMS length, cm & \\
6 & $2(3.8)$ \\
8 & $30(56.6)$ \\
10 & $21(39.6)$ \\
SEMS
\end{tabular}

SEMS diameter, $\mathrm{mm}$

Deployment technique

TTS

TTS under radiographic guidance

Total operators $(n=4)$

$<10$ procedures

0

$\geq 10$ procedures

3

$\geq 10$ procedures with ERCP experience

Training, $\mathrm{n}(\%)$

Trainee involved

No trainee appears to be sufficient and there are no reported recurrences and procedure-related complications $(16,17)$. Given that this technique is easy to perform, does not require a dedicated device and is cost-effective, additional studies are required to determine whether this technique may serve as an alternative method for relieving acute obstruction caused by benign colonic strictures.

Patients with rectal foreign bodies have usually inserted them intentionally and request their removal. Radiological examinations, such as plain abdominal X-ray or abdominal CT scan, are routinely obtained at Tongji Hospital, which may reveal the cause of the obstruction. However, the value of imaging studies for an impacted foreign body and fecal obstruction appears to be questionable based on our experience, since they are often negative. Regardless, the role of imaging studies is crucial for determining the inflammatory reaction in and around the bowel wall and for excluding conditions requiring surgical intervention. Following complete work-up, the patients are examined by colonoscopy. Conventional instruments were used for endoscopic foreign body extraction, such as polypectomy snare, alligator and rat tooth forceps. Endoscopy revealed no underlying pathology in the 3 patients with foreign body impaction, apart from 1 patient who had previously undergone sigmoid colectomy and a piece of surgical gauze was retained in the distal loop.

The outcome of stenting for managing colorectal obstruction from extrinsic invasive tumors has been previously investigated, although its safety and efficiency are controversial $(11,18)$. These patients often present with a complex stricture of the lumen, potentially at more than one locations, with complex adhesions, which result in bowel immobilization and altogether may contribute to the low success and high complication rate of SEMS placement. Therefore, in an elderly patient who had advanced peritoneal cancer leading to the extrinsic compression and obstruction of the lumen, a transanal colonic decompression tube was placed in the sigmoid colon as palliative therapy.

In the recent guidelines $(6,7)$, the use of SEMS as a bridge to elective surgery is not recommended for patients with curable left-sided colorectal malignant obstruction. One reason is that stent appears to adversely affect the oncological safety, without a reduction in postoperative mortality, whereas the technical and clinical success rates for stenting were lower than expected. It is suggested that the procedure may be considered to be an acceptable alternative treatment option in patients aged $>70$ years and/or with an American Society of Anesthesiologists (ASA) score of $\geq$ III $(6,7)$. However, as recognized by several other studies through comparing preoperative SEMS with emergency surgery, the use of SEMS in the acute phase has the definite advantage: It allows for rapid decompression of the colon, gaining valuable time to stabilize the patient's clinical condition and design an optimal treatment plan, overall resulting in higher successful primary anastomosis and lower stoma rates, without a significant difference in terms of complications or mortality $(8,19-23)$.

In practice, it appears that the decision to place a SEMS or operate should be made by joint consultation between patients, gastroenterologists and surgeons, with the risks and benefits weighed carefully. At our Unit, SEMS placement mainly results from a long-term follow-up (median, 35.5 months), suggesting that one session of electrocautery treatment patients with strictures have already undergone repeated dilations at local smaller-sized hospitals, but without improvement. Thus, searching for an alternative treatment option, surgery at our hospital was favored. Recent studies also highlighted the fact that electrocautery dilation is a safe and effective treatment for relieving obstruction caused by postsurgical strictures. Several studies reported promising 
serves as a bridge to colorectal cancer surgery (Table II). There was an interval of 3-7 days following stenting, which allowed for staging and systemic evaluation of the candidates' fitness for surgical resection. A total of 8 patients were found to have extensive multiple metastases; thus, stenting was used as permanent palliative therapy. The remaining 44 patients achieved one-stage surgery.

To achieve a better surgical outcome, it is crucial to improve the safety and efficiency of the stenting procedure. The main reason for technical failure of stenting is the inability to pass through the stenosis with a guidewire or the deployment system due to the severity of the obstruction or its angularity $(8,15)$. It has been suggested that SEMS should be placed by an experienced gastroenterologist, such as one who has independently placed at least $10-20$ stents $(7-9,24)$. In our Institution, four skilled operators meet that standard for performing SEMS, including an ERCP-specialized endoscopist. The technical and clinical success rates were 98 and $100 \%$, respectively. SEMS was also successfully implanted in the transverse colon in 1 case (Table II). Correct placement of the guidewire beyond the stricture is crucial for safe SEMS insertion. In our study, the TTS approach was accomplished with the assistance of a thin endoscope. This small-caliber endoscope (GIF XP260; Olympus) proved to be useful for transverse severe strictures and, hence, allowed placement of the guidewire without fluoroscopy. Only in 1 case with near complete obstruction of the descending colon, stent placement was performed under radiographic image monitoring and endoscopic guidance (Fig. 3). The procedure was performed by a skilled endoscopist with therapeutic ERCP experience (264 procedures per year). It has been well-established that $\mathrm{CO}_{2}$ as a contrast agent is safe and efficient for guiding stent placement (12). $\mathrm{CO}_{2}$ insufflation was applied at the time of the procedure and no complications were observed post-procedure. Our experience may also highlight the importance of the skills that therapeutic ERCP endoscopists have attained in traversing complex strictures, understanding radiographs and deploying stents (7).

Of note, the placement of SEMS has additional risks to be considered in order to achieving maximal safety, such as the diameter and the length of the stent, which may affect technical success and complication rates (12). There are reports that stents $<25 \mathrm{~mm}$ in diameter are associated with increased migration and those $>25 \mathrm{~mm}$ with higher perforation rates (8). A stent $20 \mathrm{~mm}$ in diameter was selected, which fits through the working channel of a thin endoscope. The length of SEMSs used was 6,8 and $10 \mathrm{~cm}$. It is crucial to measure the tumor size while passing a guidewire. Subsequently, according to the length of the tumor, a stent length of at least $2 \mathrm{~cm}$ longer compared with that of the stricture was selected. In addition, the TTS approach allows for accurate SEMS positioning under direct vision, aligning the upper end of the stent beyond the tumor margin for $\sim 1 \mathrm{~cm}$. In this manner, no migration or perforation was observed in any of the cases.

The retrospective analysis of the data indicates certain limitations, including different operators with variable experience and, most importantly, the follow-up was not recorded for those patients with SEMS placement followed by surgery. However, in our opinion, urgent colonoscopy as a minimally invasive approach has its own advantages in managing acute colorectal obstruction. Furthermore, active discussion should be fostered among the emergency, surgery/trauma and endoscopy departments to determine the optimal option for individual patients.

\section{Acknowledgements}

The authors would like to thank all the staff at the Endoscopy Unit of Tongji Hospital for their contributions. We would especially like to thank Mrs. Ji-Feng Hu, Mrs. Ming Zhang and Mrs. Qing Zhou for collecting the clinical data for this manuscript. This study was supported by the National Natural Science Foundation of China (grant no. 81471612).

\section{References}

1. ASGE Standards of Practice Committee; Harrison ME, Anderson MA, Appalaneni V, Banerjee S, Ben-Menachem T, Cash BD, Fanelli RD, Fisher L, Fukami N, et al: The role of endoscopy in the management of patients with known and suspected colonic obstruction and pseudo-obstruction. Gastrointest Endosc 71: 669-679, 2010.

2. Olmi S, Scaini A, Cesana G, Dinelli M, Lomazzi A and Croce E: Acute colonic obstruction: Endoscopic stenting and laparoscopic resection. Surg Endosc 21: 2100-2104, 2007.

3. Muller KE, Arató A, Lakatos PL, Papp M and Veres G: Foreign body impaction in the sigmoid colon: A twenty euro bet. World J Gastroenterol 19: 3892-3894, 2013.

4. Singaporewalla RM, Tan DE and Tan TK: Use of endoscopic snare to extract a large rectosigmoid foreign body with review of literature. Surg Laparosc Endosc Percutan Tech 17: 145-148, 2007.

5. Araghizadeh F: Fecal impaction. Clin Colon Rectal Surg 18: 116-119, 2005.

6. Cetinkaya E, Dogrul AB and Tirnaksiz MB: Role of self expandable stents in management of colorectal cancers. World $\mathbf{J}$ Gastrointest Oncol 8: 113-120, 2016.

7. van Hooft JE, van Halsema EE, Vanbiervliet G, Beets-Tan RG, DeWitt JM, Donnellan F, Dumonceau JM, Glynne-Jones RG, Hassan C, Jimenez-Perez J, et al: Self-expandable metal stents for obstructing colonic and extracolonic cancer: European Society of gastrointestinal endoscopy (ESGE) clinical guideline. Endoscopy 46: 990-1053, 2014.

8. Geraghty J, Sarkar S, Cox T, Lai S, Willert R, Ramesh J, Bodger K and Carlson GL: Management of large bowel obstruction with self-expanding metal stents. A multicentre retrospective study of factors determining outcome. Colorectal Dis 16: 476-483, 2014.

9. Williams D, Law R and Pullyblank AM: Colorectal stenting in malignant large bowel obstruction: The learning curve. Int J Surg Oncol 2011: 917848, 2011.

10. Repici A, Adler DG, Gibbs CM, Malesci A, Preatoni P and Baron TH: Stenting of the proximal colon in patients with malignant large bowel obstruction: Techniques and outcomes. Gastrointest Endosc 66: 940-944, 2007.

11. Keswani RN, Azar RR, Edmundowicz SA, Zhang Q, Ammar T, Banerjee B, Early DS and Jonnalagadda SS: Stenting for malignant colonic obstruction: A comparison of efficacy and complications in colonic versus extracolonic malignancy. Gastrointest Endosc 69: 675-680, 2009.

12. Baron TH, Wong Kee Song LM and Repici A: Role of self-expandable stents for patients with colon cancer (with videos). Gastrointest Endosc 75: 653-662, 2012.

13. Di Giorgio P, De Luca L, Rivellini G, Sorrentino E, D'amore E and De Luca B: Endoscopic dilation of benign colorectal anastomotic stricture after low anterior resection: A prospective comparison study of two balloon types. Gastrointest Endosc 60: 347-350, 2004.

14. Ferlitsch A, Reinisch W, Püspök A, Dejaco C, Schillinger M, Schöfl R, Pötzi R, Gangl A and Vogelsang H: Safety and efficacy of endoscopic balloon dilation for treatment of Crohn's disease strictures. Endoscopy 38: 483-487, 2006.

15. Adler DG: Colonic strictures: Dilation and stents. Gastrointest Endosc Clin N Am 25: 359-371, 2015.

16. Bravi I, Ravizza D, Fiori G, Tamayo D, Trovato C, De Roberto G, Genco $C$ and Crosta C: Endoscopic electrocautery dilation of benign anastomotic colonic strictures: A single-center experience. Surg Endosc 30: 229-232, 2016. 
17. Brandimarte G, Tursi A and Gasbarrini G: Endoscopic treatment of benign anastomotic colorectal stenosis with electrocautery. Endoscopy 32: 461-463, 2000.

18. Shin SJ, Kim TI, Kim BC, Lee YC, Song SY and Kim WH: Clinical application of self-expandable metallic stent for treatment of colorectal obstruction caused by extrinsic invasive tumors. Dis Colon Rectum 51: 578-583, 2008.

19. Ng KC, Law WL, Lee YM, Choi HK, Seto CL and Ho JW: Self-expanding metallic stent as a bridge to surgery versus emergency resection for obstructing left-sided colorectal cancer: A case-matched study. J Gastrointest Surg 10: 798-803, 2006.

20. Huang X, Lv B, Zhang S and Meng L: Preoperative colonic stents versus emergency surgery for acute left-sided malignant colonic obstruction: A meta-analysis. J Gastrointest Surg 18: 584-591, 2014.
21. Tan CJ, Dasari BV and Gardiner K: Systematic review and meta-analysis of randomized clinical trials of self-expanding metallic stents as a bridge to surgery versus emergency surgery for malignant left-sided large bowel obstruction. Br J Surg 99: 469-476, 2012

22. Occhionorelli S, Tartarini D, Cappellari L, Stano R and Vasquez G: Colonic stent placement as a bridge to surgery in patients with left-sided malignant large bowel obstruction. An observational study. G Chir 35: 283-289, 2014.

23. Sagar J: Role of colonic stents in the management of colorectal cancers. World J Gastrointest Endosc 8: 198-204, 2016.

24. Katsanos KH, Maliouki M, Tatsioni A, Ignatiadou E, Christodoulou DK, Fatouros M and Tsianos EV: The role of colonoscopy in the management of intestinal obstruction: A 20-year retrospective study. BMC Gastroenterol 10: 130, 2010 